\title{
Alkali Halide Surfaces Near Melting: Wetting and Nanofriction Properties
}

\author{
D. Ceresoli ${ }^{\mathrm{a}, \mathrm{b}}$, T. Zykova-Timan $^{\mathrm{a}, \mathrm{b}, 1}, \mathrm{U}$. Tartaglino ${ }^{\mathrm{a}, \mathrm{b}}$ and \\ E. Tosatti a,b,c,* \\ ${ }^{a}$ Scuola Internazionale Superiore di Studi Avanzati (SISSA), via Beirut 2-4, 34014 \\ Trieste, Italy \\ ${ }^{\mathrm{b}}$ DEMOCRITOS National Simulation Center, via Beirut 2-4, 34014 Trieste, Italy \\ ${ }^{\mathrm{c}}$ International Center for Theoretical Physics (ICTP), Strada Costiera 11, 34014 \\ Trieste, Italy
}

\begin{abstract}
Alkali halide (100) crystal surfaces are poorly wetted by their own melt at the triple point. We carried out simulations for $\mathrm{NaCl}(100)$ within the well tested BMHFT model potential. Calculations of the solid-vapor, solid-liquid and liquid-vapor free energies showed that solid $\mathrm{NaCl}(100)$ is a non-melting surface, and explain its bad wetting in detail. The extreme stability of $\mathrm{NaCl}(100)$ is ideal for a study of the nanofriction in the high temperature regime, close to and even above the bulk melting temperature $\left(T_{\mathrm{M}}\right)$. Our simulations reveal in this regime two distinct and opposite phenomena for plowing and for grazing friction. We found a frictional drop close to $T_{\mathrm{M}}$ for deep ploughing and wear, but on the contrary a frictional rise for grazing, wearless sliding. For both phenomena we obtain a fresh microscopic understanding, relating the former to "skating" through a local liquid cloud, the latter to softening of the free substrate surface. It is argued that both phenomena, to be pursued experimentally, should be much more general than the specific $\mathrm{NaCl}$ surface case. Most metals in particular possessing one or more close packed nonmelting surface, such as $\mathrm{Pb}, \mathrm{Al}$ or $\mathrm{Au}(111)$, should behave quite similarly.
\end{abstract}

Key words: Alkali halides, Equilibrium thermodynamics and statistical mechanics, Molecular dynamics, Surface energy, Surface melting, Wetting

\footnotetext{
* Corresponding author. Phone: +39-040-3787-438, Fax: +39-040-3787-528, Email address: tosatti@sissa.it (E. Tosatti).

1 Present address: Computational Science Department of Chemistry and Applied Biosciences, ETH Zurich USI-Campus, Via Giuseppe Buffi 13, LUI CH-6900 Lugano, Switzerland
} 


\section{Introduction}

The high temperature surface properties of alkali halide crystals, particularly of $\mathrm{NaCl}$, are very unusual as these solids are incompletely wetted by their own melt. [1] We investigated theoretically these surfaces and their uncommon behavior, with a multiple goal. The first goal was to uncover the physical reasons for the poor wetting. The second was to exploit the availability of the standard empirical potential parametrized long ago by Tosi and Fumi (BMHFT) for a first quantitative characterization of all the interfaces - and particularly their structure and their temperature dependent interface free energies - in a well defined solid-liquid-vapor system. Achieving this kind of goal is of particular interest in the general context of high temperature capillarity, a field where such a detailed understanding is usually unavailable. A third goal was to verify whether the solid $\mathrm{NaCl}(100)$ surface exhibits surface non-melting, as the partial self-wetting would imply on thermodynamic grounds. A fourth goal was to study the $\mathrm{NaCl}$ liquid surface, extracting from its temperature dependent surface tension the surface entropy, and comparing it. e.g., with the solid surface entropy at the melting point. Again, it seems that this comparison is generally unavailable. A fifth and more practical type of goal was finally to put to use the understanding obtained for the non-melting solid surface, in the field of nanofriction. In particular, our aim is to provide a first theory and simulation approach to both ploughing and grazing friction of hard nanotips on a solid surface near melting.

In the following we summarize the results of work that appeared in Refs. [2,3, 4,, 5$]$.

\section{Partial wetting of $\mathrm{NaCl}$ by its own melt}

Our classical molecular dynamics simulations with the Tosi-Fumi potentials confirm that in this model crystalline $\mathrm{NaCl}(100)$ is only partly wetted by a (nano) droplet of molten $\mathrm{NaCl}$. [2] Moreover, as expected, solid $\mathrm{NaCl}(100)$ is confirmed to be a non-melting surface, stable without any precursor signals of melting up to the bulk melting point. In a metastable state, and in the absence of defects, it can even be overheated by as much as $100 \mathrm{~K}$ above the melting temperature (Fig. 1). Extracting from the simulations the properties of the three interfaces - solid-vapor, liquid-vapor, and solid-liquid - we calculated and analyzed their free energies (Fig. 2) and found that the surface nonmelting of $\mathrm{NaCl}(100)$ and the resulting partial self-wetting stem from three separate reasons:

(i) Solid $\mathrm{NaCl}(100)$ is an exceptionally stable solid surface (Fig. 1), with a strongly decreasing surface free energy at high temperature (Fig. 2). Sta- 
bility in this regime is enhanced by extremely large anharmonicities. At the melting point $T_{\mathrm{M}}$, we found $\gamma_{\mathrm{SV}}=103 \mathrm{~mJ} / \mathrm{m}^{2}$.

(ii) The solid-liquid interface is spatially sharp (Fig. 31), and is energetically very expensive $\left(\gamma_{\mathrm{SL}} \simeq 36 \mathrm{~mJ} / \mathrm{m}^{2}\right)$. This can be attributed to large structural differences between solid and liquid, in particular a $27 \%$ density difference between the two.

(iii) The liquid-vapor interface free energy (surface tension) is relatively high $\left(\gamma_{\mathrm{LV}} \simeq 102 \mathrm{~mJ} / \mathrm{m}^{2}\right)$, actually almost identical to the solid-vapor free energy at the melting point. The reason for this high surface tension is traced to a surprising deficit of liquid surface entropy, which we calculate to be a factor $\simeq 2.5$ lower than that of the solid surface. Low entropy implies some kind of short range order at the molten salt surface. We highlighted and characterized in particular an incipient pairwise molecular charge order in the outermost regions of the molten salt surface (Fig. 4). [3]

\section{High temperature nanofriction}

The unusual stability of the alkali halide surface against melting suggests adopting it as a natural toy system for the study of high temperature AFM tip-surface nanofriction studies. A non-melting surface will not automatically liquefy under a tip, (as would instead be the case for a regular, melting surface [6]) even at temperatures very close to bulk melting. For this reason, $\mathrm{NaCl}(100)$ and other alkali halide surfaces should provide good testing grounds for a first study of high temperature nanofriction, a potentially important area which is still largely unexplored. In the absence of any experimental data on high temperature nanofriction, we conducted exploratory molecular dynamics sliding friction simulations of hard (diamond) tips on $\mathrm{NaCl}(100)$, both in the heavy ploughing, wear-dominated regime, and in the light grazing, wearless regime.

The simulated ploughing friction of a sharp penetrating tip is very large, as expected for scratching a hard solid (Fig. 5 ). However, it shows as a function of temperature a strong drop near the melting point. This high temperature friction decrease can be assimilated to skating of the tip over the hot solid. As in ice skating, the tip is surrounded by a local liquid halo, moving along with the tip as it ploughs on. [5]

At the opposite extreme, we found that simulated grazing friction of a hard flat tip over $\mathrm{NaCl}(100)$ behaves just the other way around (Fig. 6). Unlike ploughing, low temperature grazing friction is initially very small, on account of the incommensurability of the two respective lattice planes. Upon heating however the grazing friction surges just near the melting point. This rise is seen

as the analog of the celebrated peak effect in the critical current close to the 
upper critical field in the mixed state of type II superconductors. The dramatic softening of the flux lattice just before its disappearance causes a sudden increase of sliding friction of fluxons against the pinning impurities. [7,8] In our case the softening of the $\mathrm{NaCl}$ surface lattice, still solid at the melting temperature, reflects in an increase of sliding friction just before spontaneous breakdown of the solid surface above the melting point. [5]

\section{Conclusions}

This contrasting behavior of friction close to the melting point - skating when scratching, braking when grazing - should be more general than the specific instance of $\mathrm{NaCl}(100)$ where we uncovered them. Metals in particular usually possess at least one close packed non-melting surface [9] such as the (111) or the (110) surfaces of $f c c$ or bcc metals respectively, which is non-melting and should behave similarly to $\mathrm{NaCl}(100)$. We moreover can expect possible modifications of the high temperature nanofriction behavior by adhesive

forces between tip and surface, as well as by application of a bias between (conducting) tips and surfaces.

\section{Acknowledgments}

This work was sponsored by MIUR and COFIN 2006022847, as well as by INFM (Iniziativa trasversale calcolo parallelo).

\section{References}

[1] G. Grange, B. Mutaftschiev, Surf. Sci. 47 (1975) 723.

[2] T. Zykova-Timan, U. Tartaglino, D. Ceresoli, W. Sekkal-Zaoui, E. Tosatti, Surf. Sci. 566 (2004) 794.

[3] T. Zykova-Timan, D. Ceresoli, U. Tartaglino, E. Tosatti, Phys. Rev.Lett. 94 (2005) 176105.

[4] T. Zykova-Timan, D. Ceresoli, U. Tartaglino, E. Tosatti, J. Chem. Phys. 123 (2005) 164701.

[5] T. Zykova-Timan, D. Ceresoli, E. Tosatti, Nature Materials 6, 230 (2007).

[6] O. Tomagnini, F. Ercolessi, E. Tosatti, Surf. Sci. 287/288 (1993) 1041.

[7] C. Tang, X. Ling, S. Bhattacharya, P. Chaikin, Europhys. Lett 35 (1996) 597. 
[8] E. Granato, T. Ala-Nissia, S. Ying, Phys. Rev. B 62 (2000) 11834, and references therein.

[9] U. Tartaglino, T. Zykova-Timan, F. Ercolessi, E. Tosatti, Phys. Repts. 411 (2005) 291. 


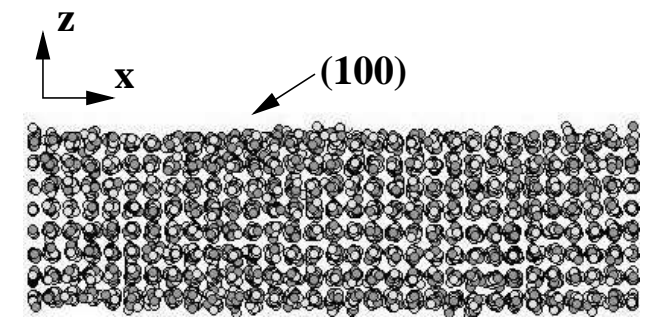

Fig. 1. Snapshot of a simulation of a $\mathrm{NaCl}(100)$ slab, at $1120 \mathrm{~K} \simeq T_{\mathrm{M}}+50 \mathrm{~K}$ (the calculated bulk melting temperature is $T_{\mathrm{M}}=1066 \mathrm{~K}$, in good agreement with experimental data). The (100) surface is normal to the $z$ direction, indicated in the figure. In our simulations at $1120 \mathrm{~K}, \mathrm{NaCl}(100)$ remains crystalline in a metastable state for at least $200 \mathrm{ps,} \mathrm{confirming} \mathrm{its} \mathrm{non-melting} \mathrm{nature.} \mathrm{Upon} \mathrm{in-}$ creasing temperature, crystalline $\mathrm{NaCl}(100)$ persists up to a surface spinodal temperature $T_{\mathrm{ss}} \simeq 1215 \mathrm{~K} \simeq T_{\mathrm{M}}+150 \mathrm{~K}$.
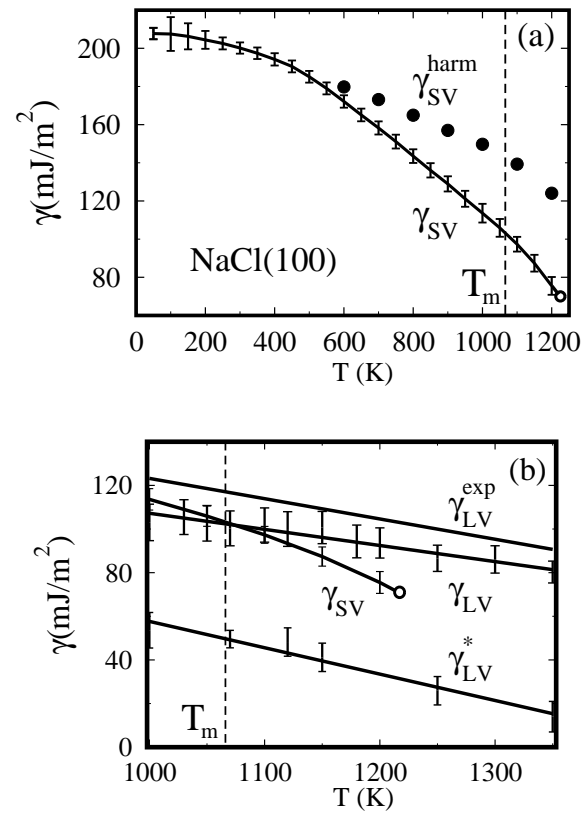

Fig. 2. (a) The solid surface free energy $\gamma_{\mathrm{SV}}$ from thermodynamic integration (solid line) and from the effective harmonic approximation (dots) [4]. (b) Liquid $\mathrm{NaCl}$ surface tension $\gamma_{\mathrm{LV}}$, compared to the experimental value $\gamma_{\mathrm{LV}}^{\exp }$. The solid surface free energy $\gamma_{\mathrm{SV}}$ is reported in the same plot. $\gamma_{\mathrm{LV}}^{\star}$ is the surface tension re-calculated by artificially removing the correlations between atoms at the outer surface. [34] Once surface molecular order is removed in this way, the surface entropy rises and the surface tension drops. Solid $\mathrm{NaCl}(100)$ would be completely wet by this artificial liquid. 


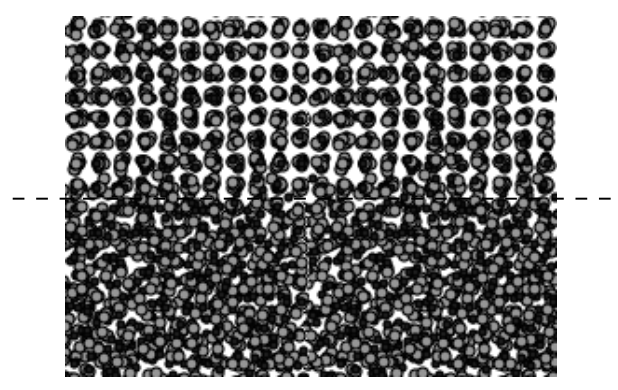

Fig. 3. Simulated $\mathrm{NaCl}(100)$ solid-liquid interface at the melting point. The interface is quite sharp. Its free energy is calculated to be $\gamma_{\mathrm{SL}}=36 \pm 5 \mathrm{~mJ} / \mathrm{m}^{2}$.

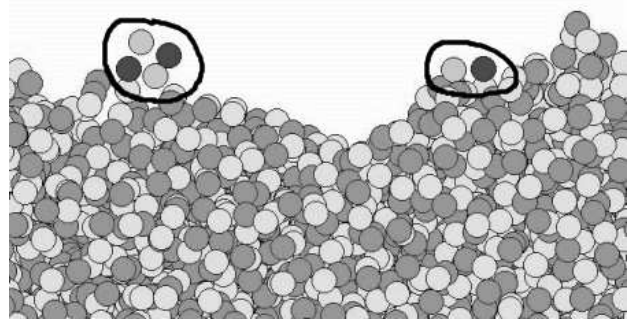

Fig. 4. Snapshot of a simulation of a liquid $\mathrm{NaCl}$ surface (liquid-vapor interface), at $1250 \mathrm{~K}$ well above the melting point and even above the surface spinodal temperature $T_{\mathrm{ss}}$. Note the very pronounced fluctuations in the instantaneous surface profile. This picture, suggestive of a low surface tension, high entropy surface, is in apparent contradiction with the massive non-wetting of solid $\mathrm{NaCl}(100)$. Indeed, we found in simulation [34, an incipient molecular surface ordering which lowers the surface entropy by a factor $\simeq 2.5$. The origin of this molecular surface order is charge order, which, already important in bulk, plays an enhanced role at the molecular liquid surface. Analyzing the correlations between $\mathrm{Na}^{+}$and $\mathrm{Cl}^{-}$ions at the surface, one finds that the outermost layer is rich in $\mathrm{NaCl}$ monomers and dimers, as highlighted in the figure. 


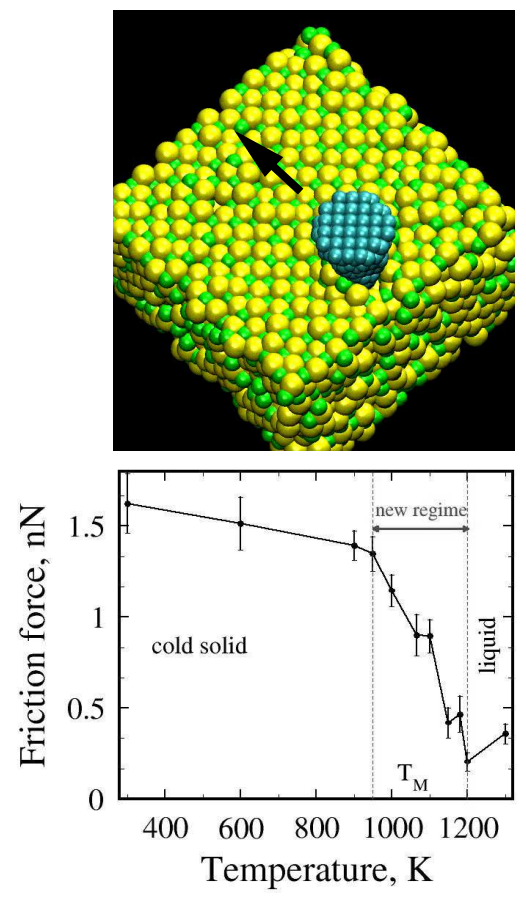

Fig. 5. Top panel: initial stage picture of ploughing friction of a hard, sharp tip on $\mathrm{NaCl}(100), 100$ degrees below the melting point. The tip is modeled as a rigid diamond apex, $26 \AA$ long. The tip indentation depth is $\simeq 6 \AA$. Note the fast-healing furrow behind the tip. Bottom panel: averaged ploughing frictional force $\left\langle F_{x}\right\rangle$ as a function of temperature. Note the friction drop at around $T=T_{\mathrm{M}}-150 \mathrm{~K}$, which is due to "skating" of the tip. 


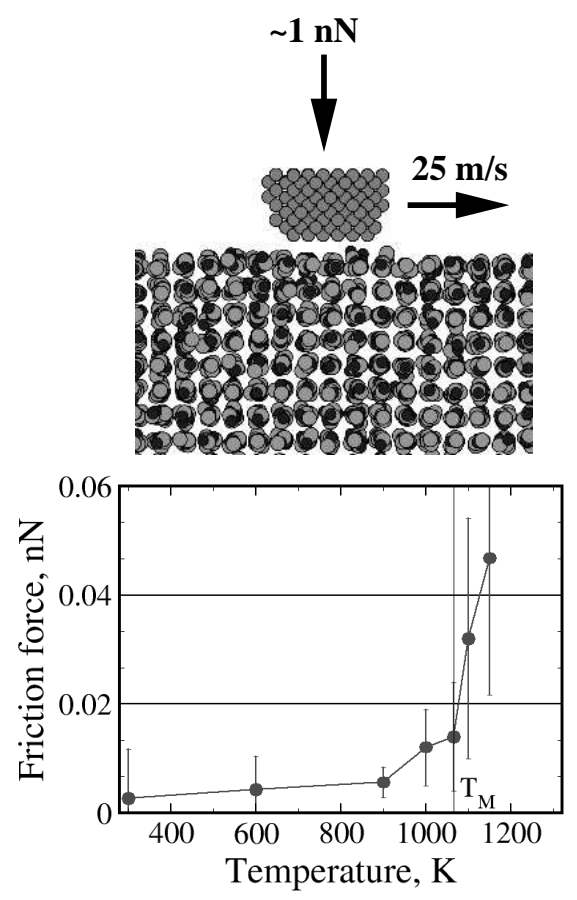

Fig. 6. Top panel: snapshot a grazing friction simulation. The tip is modeled as rigid hard diamond tip, exposing a flat facet of about $132 \AA^{2}$. The tip contacts the substrate by mere adhesion forces, which we estimate to be $\simeq 1 \mathrm{nN}$. Bottom panel: average frictional force of the grazing tip on $\mathrm{NaCl}(100)$. Note the initially very low friction (due to incommensurability between the two lattices), however sharply increasing at $T_{\mathrm{M}}$ and above. Note the contrast with the ploughing friction drop of Fig. 5 